\title{
STABILITY AND STABILIZABILITY OF DYNAMICAL SYSTEMS WITH MULTIPLE TIME-VARYING DELAYS: DELAY-DEPENDENT CRITERIA
}

\author{
D. MEHDI AND E. K. BOUKAS
}

Received 13 June 2002 and in revised form 13 January 2003

This paper deals with the class of uncertain systems with multiple time delays. The stability and stabilizability of this class of systems are considered. Their robustness are also studied when the norm-bounded uncertainties are considered. Linear matrix inequalities (LMIs) delay-dependent sufficient conditions for both stability and stabilizability and their robustness are established to check if a system of this class is stable and/or is stabilizable. Some numerical examples are provided to show the usefulness of the proposed results.

\section{Introduction}

It was shown in different studies that the presence of the time delay in the systems dynamics is the primary cause of instability and performance degradation. The class of dynamical systems with time delay has in fact attracted a lot of researchers, mainly from the control community. Many results on this class of systems have been reported in the literature. We refer the reader to $[1,7]$ and the references therein for more information.

In the present literature, there exist two techniques that can be used to study the stability and the stabilizability. The first one is based on the Lyapunov-Razumikhin technique and it consists of considering a Lyapunov function of the form $V\left(x_{t}\right)=x_{t}^{\top} P x_{t}$, with $P$ a symmetric and positive-definite matrix with appropriate dimension and $x_{t}$ the state vector of the system, to develop the conditions that can be used to check if the system under study is stable and/or stabilizable. This technique gives a condition that depends on the maximum value of the delay. The reader can consult $[2,3,4,5,7,8,10,11,12,13,14,15]$ and the references therein for more information.

The second technique is based on the Lyapunov-Krasovskii approach and it consists of considering a more complicated Lyapunov functional to determine the appropriate delaydependent condition that in general depends on the upper bound of the first derivative of the delay when it is time-varying. This technique has been extensively used and the large number of references using it confirms this. See, for example, $[1,7]$ and the references therein for more information. 
But from the practical point of view, we are interested in conditions that depend on both, that is, the upper bound of the delay and the lower and the upper bounds of the first derivative of the time-varying delay. Since in practice the delay is in fact always timevarying, that can be usually represented by a function $h(t)$ and bounded by a constant $\bar{h}$, it is therefore desirable to have conditions that depend on the upper bound of the time-varying delay and on the lower and the upper bounds of the first derivative of the time-varying delay.

The goal of this paper consists of considering the class of uncertain linear systems with multiple time-varying delays and developing sufficient conditions for stability and stabilizability and their robustness that depend on the upper bounds of the delays and on the lower and upper bounds of the first derivative of the time-varying delays. The Lyapunov-Krasovskii approach is used in this paper.

Further, the proposed state feedback controller is obtained from some LMI conditions. The LMI problem we deal with introduces some slack variables in addition to the design of a state feedback controller. These slack variables, especially in the uncertain case, give more flexibility resulting in larger bounds.

The paper is organized as follows. In Section 2, the problem is stated and the required assumptions are formulated. Section 3 deals with the stability and the robust stability. Section 4 covers the stabilizability and the robust stabilizability of the class of systems under study. Section 5 presents some numerical examples to show the usefulness of the proposed results.

Notation 1.1. In the rest of this paper, the notation is standard unless it is specified otherwise. The notation $L>0(L<0)$ means that the matrix $L$ is symmetric and positivedefinite (symmetric and negative-definite) matrix and $\operatorname{Sym}(M)=M+M^{\top}$.

\section{Problem statement}

We consider the following class of systems with multiple time-varying delays:

$$
\dot{x}_{t}=A(t) x_{t}+\sum_{j=1}^{p} A_{d j}(t) x_{t-h_{j}(t)}+B(t) u_{t},
$$

where $x_{t}$ is the state vector, $u_{t}$ is the control input, $h_{j}(t), j=1,2, \ldots, p$, is the time-varying delay of the system and the matrices $A(t), A_{d j}(t)$, and $B(t)$ are given by

$$
\begin{aligned}
A(t) & =A+D F(t) E, \\
A_{d j}(t) & =A_{d j}+D_{j} F_{j}(t) E_{j}, \quad \forall j=1,2, \ldots, p, \\
B(t) & =B+D_{b} F_{b}(t) E_{b},
\end{aligned}
$$

with $A, A_{d j}, j=1,2, \ldots, p, B, D, E, D_{j}, E_{j}, j=1,2, \ldots, p, D_{b}$, and $E_{b}$ given matrices with appropriate dimensions and $F(t), F_{j}(t), j=1,2, \ldots, p$, and $F_{b}(t)$ representing the system uncertainties satisfying the following assumption. 
Assumption 2.1. We assume that the following hold:

$$
F^{\top}(t) R F(t) \leq R, \quad F_{d}^{\top}(t) R_{d} F_{d}(t) \leq R_{d}, \quad F_{b}^{\top}(t) R_{b} F_{b}(t) \leq R_{b}
$$

where $R_{d}(t)$ and $F_{d}(t)$ are diagonal matrices given by

$$
F_{d}(t)=\left[\begin{array}{lll}
F_{1}(t) & & \\
& \ddots & \\
& & F_{p}(t)
\end{array}\right], \quad R_{d}(t)=\left[\begin{array}{lll}
R_{1} & & \\
& \ddots & \\
& & R_{p}
\end{array}\right],
$$

with $R, R_{1}, \ldots, R_{p}$, and $R_{b}$ given matrices with appropriate dimensions.

Remark 2.2. The uncertainties that satisfy (2.3) will be referred to as admissible uncertainties. Notice that the uncertainties $F(t), F_{j}(t), j=1,2, \ldots, p$, and $F_{b}(t)$ can be chosen dependent on the system state and the developed results will remain valid. However, in the present paper, we will consider only the case of time-varying uncertainties.

Assumption 2.3. For each $j=1,2, \ldots, p$, the time-varying delay $h_{j}(t)$ is assumed to satisfy

$$
0 \leq h_{j}(t) \leq \bar{h}_{j}<\infty, \quad \underline{l}_{j} \leq \dot{h}_{j}(t) \leq \bar{l}_{j}<1,
$$

where $\bar{h}_{j}, \underline{l}_{j}$, and $\bar{l}_{j}$ are given positive constants.

We define $\bar{\tau}$ as $\bar{\tau}=\max \left(\bar{h}_{1}, \ldots, \bar{h}_{p}\right)$ and $\mathbf{x}_{t}$ as $\mathbf{x}_{t}(s)=x_{t+s}, t-\bar{\tau} \leq s \leq t$. In the rest of the paper, we will use $\mathbf{x}_{t}$ instead of $\mathbf{x}_{t}(s)$.

In our investigation of a robust controller, we need to bound some uncertain terms to derive sufficient conditions for robust stability or stabilizability, and Lemma 2.4 below will be very useful for this purpose.

Lemma 2.4 [6]. Let $Z, E, F, R$, and $\Delta$ be matrices of appropriate dimensions. Assume that $Z$ is symmetric, $R$ is symmetric and positive definite, and $\Delta^{\top} R \Delta \leq R$. Then

$$
Z+E \Delta F+F^{\top} \Delta^{\top} E^{\top}<0
$$

if and only if there exists a scalar $\lambda>0$ satisfying

$$
Z+E(\lambda R) E^{\top}+F^{\top}(\lambda R)^{-1} F<0
$$

In the next section, we deal with the stability problem and its robustness and establish LMI conditions that we can use to check if a system of the class we are studying is stable. 


\section{Stability and robust stability}

The goal of this section consists of establishing what will be the sufficient conditions that can be used to check if the class of systems under study is stable. We are also interested in the robust stability of this class of systems. These two problems will be discussed in the following subsections.

3.1. Stability. We now suppose that the control is equal to zero, that is, $u_{t}=0$, for all $t \geq 0$, and that the system does not contain uncertainties, a matter which gives the following dynamics:

$$
\dot{x}_{t}=A x_{t}+\sum_{j=1}^{p} A_{d j} x_{t-h_{j}(t)} .
$$

The goal of this subsection consists of developing a condition that can be used to check if the class of systems under study is stable. The condition we are looking for should depend on the upper bound of the delay and on the lower and upper bounds of the first derivative of the time-varying delays given in Assumption 2.3. The following theorem states such result.

Theorem 3.1. Assume that Assumption 2.3 is satisfied. If there exist $F_{j}, j=1,2,3,4, P>0$, $Q_{j}>0, W_{j}>0, X_{j}, Y_{j}$, and $Z_{j}$, for $j=1,2, \ldots, p$, such that the following hold:

$$
\begin{gathered}
\mathscr{L}_{j}=\left[\begin{array}{cc}
Z_{j} & Y_{j} \\
Y_{j}^{\top} & X_{j}
\end{array}\right]>0, \\
\left(\bar{l}_{j}-\underline{l}_{j}\right) X_{j}+\left(\bar{l}_{j}-1\right) W_{j}<0, \\
{\left[\begin{array}{cccc}
\Psi_{1} & -\Psi_{3} & 0 & P \\
-\Psi_{3}^{\top} & -\Psi_{2} & 0 & 0 \\
0 & 0 & -\mathscr{W} & \mathscr{W}^{\top} \\
P & 0 & \mathbb{I}^{\mathcal{W}} & 0
\end{array}\right]+\operatorname{Sym}\left\{\left[\begin{array}{l}
F_{1} \\
F_{2} \\
F_{3} \\
F_{4}
\end{array}\right]\left[\begin{array}{llll}
A & A_{d} & 0 & -I
\end{array}\right]\right\}<,}
\end{gathered}
$$

where

$$
\begin{aligned}
\mathbb{I} & =\left[\begin{array}{lll}
I & \cdots & I
\end{array}\right], \\
A_{d} & =\left[\begin{array}{lll}
A_{d 1} & \cdots & A_{d p}
\end{array}\right], \\
\mathcal{W} & =\operatorname{diag}\left(h_{1} W_{1}, \ldots, h_{p} W_{p}\right), \\
\Psi_{1} & =\sum_{j=1}^{p}\left[Q_{j}+\left(\bar{l}_{j}-\underline{l}_{j}\right)\left(\bar{h}_{j} Z_{j}+Y_{j}+Y_{j}^{\top}\right)\right], \\
\Psi_{3} & =\left[\left(\bar{l}_{1}-\underline{l}_{1}\right) Y_{1} \cdots\left(\bar{l}_{p}-\underline{l}_{p}\right) Y_{p}\right], \\
\Psi_{2} & =\operatorname{diag}\left(\left(1-\bar{l}_{1}\right) Q_{1}, \ldots,\left(1-\bar{l}_{p}\right) Q_{p}\right),
\end{aligned}
$$

then the system under study is asymptotically stable. 
In order to prove Theorem 3.1, we give the following lemma.

Lemma 3.2. Condition (3.4) is equivalent to

$$
\left[\begin{array}{ccc}
A^{\top} P+P A+\Psi_{1} & P A_{d}-\Psi_{3} & A^{\top} \mathbb{I}^{\mathcal{W}} \\
\left(P A_{d}-\Psi_{3}\right)^{\top} & -\Psi_{2} & A_{d}^{\top} \mathbb{I}^{\mathcal{W}} \\
\mathcal{W} \mathbb{I}^{\top} A & W \mathbb{I}^{\top} A_{d} & -\mathscr{W}
\end{array}\right]<0
$$

Proof. The proof is a direct application of the elimination lemma; that is, notice that

$$
\mathcal{N}_{\text {right }}=\left[\begin{array}{ccc}
I & 0 & 0 \\
0 & I & 0 \\
0 & 0 & I \\
A & A_{d} & 0
\end{array}\right]
$$

satisfies

$$
\left[\begin{array}{cccc}
A & A_{d} & 0 & -I
\end{array}\right]\left[\begin{array}{ccc}
I & 0 & 0 \\
0 & I & 0 \\
0 & 0 & I \\
A & A_{d} & 0
\end{array}\right]=0
$$

Then condition (3.4) is equivalent to

$$
\mathcal{N}_{\text {right }}^{\top}\left[\begin{array}{cccc}
\Psi_{1} & -\Psi_{3} & 0 & P \\
-\Psi_{3}^{\top} & -\Psi_{2} & 0 & 0 \\
0 & 0 & -\mathcal{W} & \mathcal{W I}^{\top} \\
P & 0 & \mathbb{I}^{\top} W & 0
\end{array}\right] \mathcal{N}_{\text {right }}=\left[\begin{array}{ccc}
A^{\top} P+P A+\Psi_{1} & P A_{d}-\Psi_{3} & A^{\top} \mathbb{I}^{\mathscr{W}} \\
\left(P A_{d}-\Psi_{3}\right)^{\top} & -\Psi_{2} & A_{d}^{\top} \mathbb{I}^{\mathcal{W}} \\
\mathcal{W} \mathbb{I}^{\top} A & \mathcal{W} \mathbb{I}^{\top} A_{d} & -\mathscr{W}
\end{array}\right]<0
$$

and this ends the proof of Lemma 3.2.

Proof of Theorem 3.1. Let the Lyapunov functional be defined by

$$
V\left(\mathbf{x}_{t}\right)=V_{1}\left(\mathbf{x}_{t}\right)+V_{2}\left(\mathbf{x}_{t}\right)+V_{3}\left(\mathbf{x}_{t}\right)+V_{4}\left(\mathbf{x}_{t}\right),
$$


where

$$
\begin{aligned}
& V_{1}\left(\mathbf{x}_{t}\right)=x_{t}^{\top} P x_{t}, \\
& V_{2}\left(\mathbf{x}_{t}\right)=\sum_{j=1}^{p} \int_{t-h_{j}(t)}^{t} \int_{s}^{t} \dot{x}_{z}^{\top} W_{j} \dot{x}_{z} d z d s, \\
& V_{3}\left(\mathbf{x}_{t}\right)=\sum_{j=1}^{p} \int_{t-h_{j}(t)}^{t} x_{s}^{\top} Q_{j} x_{s} d s, \\
& V_{4}\left(\mathbf{x}_{t}\right)=\sum_{j=1}^{p} \int_{0}^{t}\left(\bar{l}_{j}-\dot{h}_{j}(z)\right) \int_{z-h_{j}(z)}^{z}\left[\begin{array}{ll}
x_{z}^{\top} & \dot{x}_{s}
\end{array}\right]\left[\begin{array}{cc}
Z_{j} & Y_{j} \\
Y_{j}^{\top} & X_{j}
\end{array}\right]\left[\begin{array}{c}
x_{z} \\
\dot{x}_{s}
\end{array}\right] d s d z .
\end{aligned}
$$

After taking the derivative of these functionals and some algebraic manipulations, we get

$$
\dot{V}\left(\mathbf{x}_{\mathbf{t}}\right)=\xi_{t}^{\top} M \xi_{t}+\sum_{j=1}^{p} \int_{t-h_{j}(t)}^{t} \dot{x}_{s}^{\top}\left[\left(\bar{l}_{j}-\underline{l}_{j}\right) X_{j}+\left(\bar{l}_{j}-1\right) W_{j}\right] \dot{x}_{s} d s,
$$

with

$$
\begin{aligned}
\xi_{t}^{\top} & =\left[\begin{array}{lll}
x_{t}^{\top} x_{t-h_{1}(t)}^{\top} & \cdots & x_{t-h_{p}(t)}^{\top}
\end{array}\right], \\
M & =\left[\begin{array}{ll}
M_{11} & M_{12} \\
M_{12}^{\top} & M_{22}
\end{array}\right],
\end{aligned}
$$

where $M_{11}, M_{12}$, and $M_{22}$ are given by

$$
\begin{aligned}
& M_{11}=A^{\top} P+P A+A^{\top} \mathbb{I}^{\top} W \mathbb{I}^{\top} A+\Psi_{1}, \\
& M_{12}=P A_{d}-\Psi_{3}+A^{\top} \mathbb{I}^{\alpha} W \mathbb{I}^{\top} A_{d}, \\
& M_{22}=A_{d}^{\top} \mathbb{I}^{q} W \mathbb{I}^{\top} A_{d}-\Psi_{2} .
\end{aligned}
$$

Therefore, the system is then asymptotically stable if the following hold:

$$
\begin{gathered}
M<0, \\
{\left[\left(\bar{l}_{j}-\underline{l}_{j}\right) X_{j}+\left(\bar{l}_{j}-1\right) W_{j}\right]<0, \quad \forall j=1, \ldots, p .}
\end{gathered}
$$

Notice that matrix $M$ can be expressed as follows:

$$
\begin{aligned}
& M=\left[\begin{array}{cc}
A^{\top} P+P A+\Psi_{1} & P A_{d}-\Psi_{3} \\
\left(P A_{d}-\Psi_{3}\right)^{\top} & -\Psi_{2}
\end{array}\right]+\left[\begin{array}{cc}
A^{\top} \mathbb{I}^{\top} W \mathbb{I}^{\top} A & A^{\top} \mathbb{I}^{\top} W \mathbb{I}^{\top} A_{d} \\
\left(A^{\top} \mathbb{I}^{\top} W \mathbb{I}^{\top} A_{d}\right)^{\top} & A_{d}^{\top} \mathbb{I}^{\top} W \mathbb{I}^{\top} A_{d}
\end{array}\right] \\
& =\left[\begin{array}{cc}
A^{\top} P+P A+\Psi_{1} & P A_{d}-\Psi_{3} \\
\left(P A_{d}-\Psi_{3}\right)^{\top} & -\Psi_{2}
\end{array}\right]+\left[\begin{array}{c}
A^{\top} \mathbb{I}^{\mathcal{W}} \\
A_{d}^{\top} \mathbb{I}^{\mathcal{W}}
\end{array}\right](\mathcal{W})^{-1}\left[\begin{array}{c}
A^{\top} \mathbb{I}^{\top} \mathcal{W} \\
A_{d}^{\top} \mathbb{I}^{\top} \mathcal{W}
\end{array}\right]^{\top} \text {. }
\end{aligned}
$$


Using Schur complement, we conclude that $M$ is negative definite if and only if condition (3.6) is satisfied. Furthermore, since condition (3.6) is equivalent to (3.4) according to Lemma 3.2 and since (3.4) is verified by assumption as well as conditions (3.3) and (3.2), then the system under study is asymptotically stable. This ends the proof of the theorem.

Remark 3.3. The results of Theorem 3.1 are only sufficient and, therefore, if these conditions are not verified, we cannot claim that the system under study is not stable.

3.2. Robust stability. We now assume that the control is still equal to zero for all time and assume that the system has uncertainties on all the matrices, that is,

$$
\dot{x}_{t}=[A+D F(t) E] x_{t}+\sum_{j=1}^{p}\left[A_{d j}+D_{j} F_{j}(t) E_{j}\right] x_{t-h_{j}(t)},
$$

where all the terms keep the same meaning as before.

We introduce the following notations:

$$
\begin{aligned}
\tilde{A} & =A+D F(t) E \\
\tilde{A}_{d} & =\left[\begin{array}{lll}
A_{d 1}+D_{1} F_{1}(t) E_{1} & \cdots & A_{d p}+D_{p} F_{p}(t) E_{p}
\end{array}\right]=A_{d}+D_{d} F_{d} E_{d},
\end{aligned}
$$

where $E_{d}$ and $D_{d}$ are given by

$$
D_{d}=\left[\begin{array}{lll}
D_{1} & \cdots & D_{p}
\end{array}\right], \quad E_{d}=\operatorname{diag}\left(E_{1}, \ldots, E_{p}\right)
$$

Note that conditions (3.2) and (3.3) do not depend on the system matrices so they do not need to be adapted to the uncertain case. Besides, we have to replace $A$ and $A_{d}$ by $\tilde{A}$ and $\tilde{A}_{d}$, respectively, in condition (3.4) to get a condition for the robust case which is stated by Theorem 3.4.

Theorem 3.4. Assume that Assumptions 2.1 and 2.3 are satisfied. If there exist $F_{1}, F_{2}, F_{3}$, $F_{4}, P>0, Q_{j}>0, W_{j}>0, X_{j}, Y_{j}, Z_{j}$, for $j=1,2, \ldots, p$, and $\lambda$ such that conditions (3.2), (3.3), and

$$
\left[\begin{array}{cccccc}
\alpha_{11} & * & * & * & * & * \\
A_{d}^{T} F_{1}^{T}+F_{2} A-\Psi_{3}^{\top} & \alpha_{22} & * & * & * & * \\
F_{3} A & F_{3} A_{d} & -\mathscr{W} & * & * & * \\
F_{4} A+P-F_{1}^{\top} & F_{4} A_{d}-F_{2}^{\top} & \mathbb{I}^{\top} W-F_{3}^{\top} & -F_{4}-F_{4}^{\top} & * & * \\
D^{\top} F_{1}^{\top} & D^{\top} F_{2}^{\top} & D^{\top} F_{3}^{\top} & D^{\top} F_{4}^{\top} & -\lambda R & * \\
D_{d}^{\top} F_{1}^{\top} & D_{d}^{\top} F_{2}^{\top} & D_{d}^{\top} F_{3}^{\top} & D_{d}^{\top} F_{4}^{\top} & 0 & -\lambda R_{d}
\end{array}\right]<0
$$


hold, with

$$
\begin{aligned}
& \alpha_{11}=\Psi_{1}+A^{T} F_{1}^{T}+F_{1} A+\lambda E^{\top} R E, \\
& \alpha_{22}=A_{d}^{T} F_{2}^{T}+F_{2} A_{d}-\Psi_{2}+\lambda E_{d}^{\top} R_{d} E_{d},
\end{aligned}
$$

then the uncertain system under study is asymptotically stable for all admissible uncertainties. Proof of Theorem 3.4. As we have said before, the robust stability is achieved, according to Theorem 3.1, if conditions (3.2), (3.3), and

$$
\left[\begin{array}{cccc}
\Psi_{1} & -\Psi_{3} & 0 & P \\
-\Psi_{3}^{\top} & -\Psi_{2} & 0 & \\
0 & 0 & -\mathscr{W} & W_{\mathbb{I}^{\top}} \\
P & 0 & \mathbb{I}^{\mathcal{W}} & 0
\end{array}\right]+\operatorname{Sym}\left\{\left[\begin{array}{c}
F_{1} \\
F_{2} \\
F_{3} \\
F_{4}
\end{array}\right]\left[\begin{array}{llll}
\tilde{A} & \tilde{A}_{d} & 0 & -I
\end{array}\right]\right\}<0
$$

are satisfied. Since conditions (3.2) and (3.3) remain unchanged in the presence of uncertainty, we have to work out only condition (3.22).

First, notice that in condition (3.22) the second term of the left side can be split into two parts to yield

$$
\begin{gathered}
{\left[\begin{array}{cccc}
\Psi_{1} & -\Psi_{3} & 0 & P \\
-\Psi_{3}^{\top} & -\Psi_{2} & 0 & 0 \\
0 & 0 & -\mathcal{W} & \mathcal{W I}^{\top} \\
P & 0 & \mathbb{I}^{\top} \mathcal{W} & 0
\end{array}\right]+\operatorname{Sym}\left\{\left[\begin{array}{l}
F_{1} \\
F_{2} \\
F_{3} \\
F_{4}
\end{array}\right]\left[\begin{array}{cccc}
A & A_{d} & 0 & -I
\end{array}\right]\right\}} \\
+\operatorname{Sym}\left\{\left[\begin{array}{ll}
F_{1} D & F_{1} D_{d} \\
F_{2} D & F_{4} D_{d} \\
F_{3} D & F_{3} D_{d} \\
F_{4} D & F_{4} D_{d}
\end{array}\right]\left[\begin{array}{ll}
F(t) & \\
& F_{d}(t)
\end{array}\right]\left[\begin{array}{cccc}
E & 0 & 0 & 0 \\
0 & E_{d} & 0 & 0
\end{array}\right]\right\}<0 .
\end{gathered}
$$

According to Lemma 2.4, the previous inequality is satisfied if there exists $\lambda>0$ such that

$$
\begin{aligned}
& {\left[\begin{array}{cccc}
\Psi_{1} & -\Psi_{3} & 0 & P \\
-\Psi_{3}^{\top} & -\Psi_{2} & 0 & 0 \\
0 & 0 & -\mathscr{W} & \mathcal{W I}^{\top} \\
P & 0 & \mathbb{I}^{\mathscr{W}} & 0
\end{array}\right]+\operatorname{Sym}\left\{\left[\begin{array}{c}
F_{1} \\
F_{2} \\
F_{3} \\
F_{4}
\end{array}\right]\left[\begin{array}{llll}
A & A_{d} & 0 & -I
\end{array}\right]\right\}} \\
& +\left[\begin{array}{ll}
F_{1} D & F_{1} D_{d} \\
F_{2} D & F_{4} D_{d} \\
F_{3} D & F_{3} D_{d} \\
F_{4} D & F_{4} D_{d}
\end{array}\right]\left[\begin{array}{ll}
\lambda R & \\
& \lambda R_{d}
\end{array}\right]^{-1}\left[\begin{array}{ll}
F_{1} D & F_{1} D_{d} \\
F_{2} D & F_{4} D_{d} \\
F_{3} D & F_{3} D_{d} \\
F_{4} D & F_{4} D_{d}
\end{array}\right]^{\top} \\
& +\left[\begin{array}{cccc}
E & 0 & 0 & 0 \\
0 & E_{d} & 0 & 0
\end{array}\right]^{\top}\left[\begin{array}{cc}
\lambda R & \\
& \lambda R_{d}
\end{array}\right]\left[\begin{array}{cccc}
E & 0 & 0 & 0 \\
0 & E_{d} & 0 & 0
\end{array}\right]<0 \text {, }
\end{aligned}
$$


and using a Schur complement operation yields

$$
\left[\begin{array}{cccccc}
f_{11} & * & * & * & * & * \\
-\Psi_{3}^{\top} & f_{22} & * & * & * & * \\
0 & 0 & -\mathscr{W} & * & * & * \\
P & 0 & \mathbb{I}^{\alpha} \mathcal{W} & 0 & * & * \\
0 & 0 & 0 & 0 & -\lambda R & * \\
0 & 0 & 0 & 0 & 0 & -\lambda R_{d}
\end{array}\right]+\operatorname{Sym}\left\{\left[\begin{array}{c}
F_{1} \\
F_{2} \\
F_{3} \\
F_{4} \\
0 \\
0
\end{array}\right]\left[\begin{array}{llllll}
A & A_{d} & 0 & -I & D & D_{d}
\end{array}\right]\right\}<0
$$

with

$$
f_{11}=\Psi_{1}+\lambda E^{\top} R E, \quad f_{22}=-\Psi_{2}+\lambda E_{d}^{\top} R_{d} E_{d}
$$

It is worth noting that (3.25) can be rewritten as condition (3.20) and this ends the proof. This condition, (3.2), and (3.3) represent the sufficient conditions for robust stability of the class of systems under consideration.

The next section deals with the stabilizability and the robust stabilizability of the class of systems under study.

3.3. Stabilizability. This section deals with the stabilizability problem, and we try to design a controller that stabilizes the closed-loop system. We restrict ourselves to the class of memoryless state feedback controller.

Thus the state feedback controller is of the form

$$
u(t)=K x(t)
$$

Substituting (3.27) in the plant model and taking $A^{c l}=(A+B K)$, we get the closedloop dynamics

$$
\dot{x}_{t}=A^{c l} x_{t}+\sum_{j=1}^{p} A_{d j}(t) x_{t-h_{j}(t)} .
$$

We note that only condition (3.4) must be adapted to the stabilizability case. We replace $A$ by $A^{c l}$ in (3.4) to get

$$
M^{c l}=\left[\begin{array}{cccc}
\Psi_{1} & -\Psi_{3} & 0 & P \\
-\Psi_{3}^{\top} & -\Psi_{2} & 0 & 0 \\
0 & 0 & -\mathscr{W} & \mathcal{W} \mathbb{I}^{\top} \\
P & 0 & \mathbb{I}^{\mathscr{W}} & 0
\end{array}\right]+\operatorname{Sym}\left\{\left[\begin{array}{l}
F_{1} \\
F_{2} \\
F_{3} \\
F_{4}
\end{array}\right]\left[\begin{array}{llll}
A^{c l} & A_{d} & 0 & -I
\end{array}\right]\right\}<0
$$

The problem of robust stabilization by state feedback is stated by Theorem 3.5. 
Theorem 3.5. Assume that Assumption 2.3 is satisfied. If there exist $F_{1}, F_{2}, F_{3}, F_{4}, P>0$, $Q_{i}>0, W_{i}>0, X_{i}, Y_{i}, Z_{i}($ for $i=1, \ldots, p), L$, and $G$ such that

$$
\begin{gathered}
{\left[\begin{array}{cc}
\bar{Z}_{i} & \bar{Y}_{i} \\
\bar{Y}_{i}^{\top} & \bar{X}_{i}
\end{array}\right]>0,} \\
\left(\bar{l}_{i}-\underline{l}_{i}\right) \bar{X}_{i}+\left(\bar{l}_{i}-1\right) \bar{W}_{i}<0, \\
{\left[\begin{array}{ccccc}
\Psi_{1}+A_{o}^{T} F_{1}^{T}+F_{1} A_{o} & * & * & * & * \\
A_{d_{o}}^{T} F_{1}^{T}+F_{2} A_{o}-\Psi_{3}^{T} & A_{d_{o}}^{T} F_{2}^{T}+F_{2} A_{d_{o}}-\Psi_{2} & * & * & * \\
F_{3} A_{o} & F_{3} A_{d_{o}} & -\mathcal{W} & * & * \\
F_{4} A_{o}+P-F_{1}^{\top} & F_{4} A_{d_{o}}-F_{2}^{\top} & \mathbb{I}^{\top} W-F_{3}^{\top} & -F_{4}-F_{4}^{\top} & * \\
B^{T} F_{1}^{T}+L-G K_{o} & B^{T} F_{2}^{T}-G K_{d_{o}} & B^{T} F_{3}^{T} & B^{T} F_{4}^{T} & -G-G^{T}
\end{array}\right]<0}
\end{gathered}
$$

hold for $i=1, \ldots, p$ for given gains $K_{o}$ and $K_{d_{o}}$ that make the matrices $A_{o}=\left(A+B K_{o}\right)$ and $A_{d_{o}}=\left(A_{d}+B K_{d_{o}}\right)$ stable, then the closed loop system is asymptotically stable with the stabilizing feedback gain given by

$$
K=G^{-1} L
$$

Proof of Theorem 3.5. The closed loop matrix $A^{c l}$ can also be rewritten as

$$
\begin{aligned}
& A^{c l}=A+B K=A+B K_{o}+B\left(K-K_{o}\right)=A_{o}+B S_{o}, \\
& A_{d}=A_{d}+B K_{d_{o}}-B K_{d_{o}}=A_{d_{o}}+B S_{d_{o}}
\end{aligned}
$$

where the gain $K_{o}$ and $K_{d_{o}}$ are chosen in such a way that $A+B K_{o}$ and $A_{d}+B K_{d_{o}}$ are stable. This allows us to rewrite $\mathcal{M}^{c l}$ as

$$
\begin{aligned}
& M^{c l}=\left[\begin{array}{cccc}
\Psi_{1} & -\Psi_{3} & 0 & P \\
-\Psi_{3}^{\top} & -\Psi_{2} & 0 & 0 \\
0 & 0 & -\mathscr{W} & W \mathbb{T}^{\top} \\
P & 0 & \mathbb{I}^{\mathscr{W}} & 0
\end{array}\right] \\
& +\operatorname{Sym}\left\{\left[\begin{array}{l}
F_{1} \\
F_{2} \\
F_{3} \\
F_{4}
\end{array}\right]\left[\begin{array}{llll}
A_{o} & A_{d_{o}} & 0 & -I
\end{array}\right]\right\}+\operatorname{Sym}\left\{\left[\begin{array}{l}
F_{1} B \\
F_{2} B \\
F_{3} B \\
F_{4} B
\end{array}\right]\left[\begin{array}{llll}
S_{o} & S_{d_{o}} & 0 & 0
\end{array}\right]\right\}<0,
\end{aligned}
$$


and using similar arguments as in the proof of Lemma 3.2, we introduce a new variable $G$ to get the condition

$$
\begin{aligned}
M_{S F}= & {\left[\begin{array}{ccccc}
\Psi_{1} & -\Psi_{3} & 0 & P & 0 \\
-\Psi_{3}^{\top} & -\Psi_{2} & 0 & 0 & 0 \\
0 & 0 & -\mathcal{W} & \mathcal{W I}^{\top} & 0 \\
P & 0 & \mathbb{I}^{\mathcal{W}} & 0 & 0 \\
0 & 0 & 0 & 0 & 0
\end{array}\right] } \\
& +\operatorname{Sym}\left\{\left[\begin{array}{c}
F_{1} \\
F_{2} \\
F_{3} \\
F_{4} \\
0
\end{array}\right]\left[\begin{array}{lllll}
A_{o} & A_{d_{o}} & 0 & -I & B
\end{array}\right]\right\}+\operatorname{Sym}\left\{\left[\begin{array}{l}
0 \\
0 \\
0 \\
0 \\
I
\end{array}\right] G\left[\begin{array}{lllll}
S_{o} & S_{d_{o}} & 0 & 0 & -I
\end{array}\right]\right\}<0
\end{aligned}
$$

which is in fact condition (3.32) where we have introduced the change of variable $L=G K$. Indeed, notice that

$$
\left[\begin{array}{ccccc}
I & 0 & 0 & 0 & -S_{o}^{\top} \\
0 & I & 0 & 0 & -S_{d_{o}}^{\top} \\
0 & 0 & I & 0 & 0 \\
0 & 0 & 0 & I & 0
\end{array}\right] M_{S F}\left[\begin{array}{cccc}
I & 0 & 0 & 0 \\
0 & I & 0 & 0 \\
0 & 0 & I & 0 \\
0 & 0 & 0 & I \\
S_{o} & S_{d_{o}} & 0 & 0
\end{array}\right]=M^{c l}<0
$$

The introduction of the new variable $G$ allows us to perform a decoupling between the matrices $B$ and $S_{o}$ and hence between $B$ and the state feedback gain $K$.

3.4. Robust stabilizability. In this subsection, we are concerned with robust stabilizability of the uncertain system under the control law (3.27). The closed loop system is then given by

$$
\dot{x}_{t}=\left[A+B K+D F(t) E+D_{b} F_{b}(t) E_{b} K\right] x_{t}+\sum_{j=1}^{p}\left[A_{d j}+D_{j} F_{j}(t) E_{j}\right] x_{t-h_{j}(t)},
$$

where all the terms keep the same meaning as previously. Taking account of the uncertainties in (3.32), we get

$$
\tilde{M}_{S F}=\left[\begin{array}{ccccc}
\Psi_{1} & -\Psi_{3} & 0 & P & 0 \\
-\Psi_{3}^{\top} & -\Psi_{2} & 0 & 0 & 0 \\
0 & 0 & -\mathscr{W} & W \mathbb{I}^{\top} & 0 \\
P & 0 & \mathbb{I}^{\mathscr{W}} & 0 & 0 \\
0 & 0 & 0 & 0 & 0
\end{array}\right]+\operatorname{Sym}\left\{\left[\begin{array}{l}
0 \\
0 \\
0 \\
0 \\
I
\end{array}\right] G\left[\begin{array}{lllll}
S_{o} & S_{d_{o}} & 0 & 0 & -I
\end{array}\right]\right\}
$$


Stability and stabilizability

$$
\begin{aligned}
+\operatorname{Sym}\left\{\left[\begin{array}{c}
F_{1} \\
F_{2} \\
F_{3} \\
F_{4} \\
0
\end{array}\right]\left[\begin{array}{lllll}
\tilde{A}^{c l} & \tilde{A}_{d}^{c l} & 0 & -I & \tilde{B}
\end{array}\right]\right\} \\
=M_{S F_{o}}+\operatorname{Sym}\left\{\left[\begin{array}{c}
F_{1} \\
F_{2} \\
F_{3} \\
F_{4} \\
0
\end{array}\right]\left[\begin{array}{lllll}
D & D_{b} & D_{d} & D_{b} & D_{b}
\end{array}\right] \mathscr{F}(t)\left[\begin{array}{ccccc}
E & 0 & 0 & 0 & 0 \\
E_{b} K_{o} & 0 & 0 & 0 & 0 \\
0 & E_{b} K_{d_{o}} & 0 & 0 & 0 \\
0 & E_{d} & 0 & 0 & 0 \\
0 & 0 & 0 & 0 & E_{b}
\end{array}\right]\right\}<0,
\end{aligned}
$$

with

$$
\begin{aligned}
& \mathscr{F}(t)=\operatorname{diag}\left[\begin{array}{lllll}
F(t) & F_{b}(t) & F_{d}(t) & F_{b}(t) & F_{b}(t)
\end{array}\right], \\
& \tilde{A}^{c l}=\tilde{A}+\tilde{B} K_{o}, \\
& \tilde{A}_{d}^{c l}=\tilde{A}_{d}+\tilde{B} K_{d_{o}} \text {, }
\end{aligned}
$$

and $M_{S F_{o}}$ the part of $\tilde{M}_{S F}$ that contains only the non-uncertain terms. Using Lemma 2.4 as previously, we get

$$
\begin{aligned}
M_{S F R}=\operatorname{Sym}\left\{\left[\begin{array}{c}
F_{1} \\
F_{2} \\
F_{3} \\
F_{4} \\
0 \\
0
\end{array}\right]\left[\begin{array}{cccccccccc}
A_{o} & A_{d_{o}} & 0 & -I & B & D & D_{b} & D_{d} & D_{b} & D_{b}
\end{array}\right]\right\} \\
0 \\
0 \\
0
\end{aligned}
$$




$$
+\operatorname{Sym}\left\{\left[\begin{array}{l}
0 \\
0 \\
0 \\
0 \\
I \\
0 \\
0 \\
0 \\
0 \\
0
\end{array}\right] G\left[\begin{array}{llllllllll}
S_{o} & S_{d_{o}} & 0 & 0 & -I & 0 & 0 & 0 & 0 & 0
\end{array}\right]\right\}<0,
$$

with

$$
\begin{aligned}
& \beta_{11}=\Psi_{1}+\lambda\left(E^{\top} R E+K_{o}^{\top} E_{b}^{\top} R_{b} E_{b} K_{o}\right), \\
& \beta_{22}=-\Psi_{2}+\lambda\left(E_{d}^{\top} R_{d} E_{d}+K_{d_{o}}^{\top} E_{b}^{\top} R_{b} E_{b} K_{d_{o}}\right) .
\end{aligned}
$$

In condition (3.41), we have to proceed to the change of variable $L=G K$ and then we get an LMI problem from which when it is feasible, we get the feedback gain as

$$
K=G^{-1} L
$$

The following theorem summarizes the result of robust stability.

Theorem 3.6. Assume that Assumptions 2.1 and 2.3 are satisfied. If there exist $F_{1}, F_{2}, F_{3}$, $F_{4}, P>0, Q_{i}>0, W_{i}>0, X_{i}, Y_{i}, Z_{i}$ (for $\left.i=1, \ldots, p\right), L, G$, and $\lambda$ such that the LMI problem composed of the three conditions (3.30), (3.31), and (3.41) is feasible, the robust stabilizing state feedback gain is given by (3.43) and the uncertain closed loop system under study is asymptotically stable for the set of all admissible uncertainties.

\section{Example}

To show the usefulness of our results, we consider some numerical examples.

Example 4.1. The goal of the example is in fact to show the flexibility introduced by the slack variables $F_{i}, i=1, \ldots, 4$, in condition (3.4) in comparison with the LMI problem using condition (3.6) as in [9].

For this purpose, we consider that the system under study has one time delay and we solve the LMI problem resulting from Theorem 3.4.

Thus, we assume that the dynamics are described by [9]

$$
\dot{x}(t)=(A+D F(t) E) x(t)+\left(A_{d}+D_{d} F_{d}(t) E_{d}\right) x(t-h(t)),
$$

where $F(t)$ and $F_{d}(t)$ are uncertain matrices of appropriate dimension satisfying condition (2.3). 
Table 4.1

\begin{tabular}{ccc}
\hline$\dot{h}(t)$ & Maximal value of $\bar{h}[9]$ & Maximal value of $\bar{h}$ (Theorem 3.4) \\
\hline$\dot{h}(t) \leq 0.9$ & 0.1621 & 0.225 \\
$\dot{h}(t) \leq 0.8$ & 0.3802 & 0.49 \\
$\dot{h}(t) \leq 0.6$ & 1.0662 & 1.425 \\
$\dot{h}(t) \leq 0.4$ & 7.1784 & $\infty$ \\
\hline
\end{tabular}

The system matrices are as follows:

$$
\begin{gathered}
A=\left[\begin{array}{cc}
-3 & 1 \\
1 & -1
\end{array}\right], \quad A_{d}=\left[\begin{array}{cc}
-0.2 & 0.1 \\
-0.3 & -0.1
\end{array}\right], \\
D=D_{d}=0.2 I, \quad E=E_{d}=I, \quad R=R_{d}=I .
\end{gathered}
$$

The delay satisfies

$$
0<h(t) \leq \bar{h}
$$

where the bound $\bar{h}$ is obtained as the maximal value yielding feasibility for the LMI problem associated with Theorem 3.4. This maximal value is a function of the upper bound of the first derivative of the delay as shown in Table 4.1.

In this example, we proceed to a comparison with the result given in [9] and we show that in the robust case, Theorem 3.4 of the present paper provides better results than [9, Theorem 3.2]. Notice that for the case where the first derivative of the delay satisfies the condition $\dot{h}(t) \leq 0.4$, Theorem 3.4 says that the system is robustly stable for unbounded delay (a very large value) whereas in [9], the maximal value was $\bar{h}=7.1884$.

It is worth noting that for the nominal system we get similar bounds.

Example 4.2. In this example, we consider the robust stabilizability problem. For this purpose, we consider the single time-varying delay system:

$$
\dot{x}(t)=(A+D F(t) E) x(t)+\left(A_{d}+D_{d} F_{d}(t) E_{d}\right) x(t-h(t))+\left(B+D_{b} F_{b}(t) E_{b}\right) u(t),
$$

where $F(t), F_{d}(t)$, and $F_{b}(t)$ are uncertain matrices of appropriate dimension satisfying condition (2.3), with

$$
\begin{aligned}
& A=\left[\begin{array}{ll}
2.0 & 0.0 \\
1.0 & 3.0
\end{array}\right], \quad D=0.2 I, \quad E=I, \quad R=I, \\
& B=\left[\begin{array}{ll}
1.0 & 2.0 \\
1.0 & 0.0
\end{array}\right], \quad D_{b}=0.2 I, \quad E_{b}=I, \quad R_{b}=I, \\
& A_{d}=\left[\begin{array}{cc}
-0.1 & 0.0 \\
-0.8 & -1.0
\end{array}\right], \quad D_{d}=0.2 I, \quad E_{d}=I, \quad R_{d}=I .
\end{aligned}
$$


The characteristics of the first derivative of the delay are as follows:

$$
\underline{l}=0.0, \quad \bar{l}=0.825 \text {. }
$$

The application of Theorem 3.6 leads to the following results:

$$
\begin{aligned}
X & =\left[\begin{array}{cc}
30.1136 & -3.9990 \\
-3.9990 & 0.5311
\end{array}\right], & Y & =\left[\begin{array}{cc}
-3.3436 & 0.4440 \\
4.4596 & -0.5922
\end{array}\right], \\
Z & =\left[\begin{array}{cc}
0.3712 & -0.4950 \\
-0.4950 & 0.6626
\end{array}\right], & W & =\left[\begin{array}{cc}
141.9641 & -18.8526 \\
-18.8526 & 2.5036
\end{array}\right], \\
P & =\left[\begin{array}{cc}
8999.5211 & -28.5684 \\
-28.5684 & 37679.0852
\end{array}\right], & Q & =\left[\begin{array}{cc}
7942.9421 & 17142.9757 \\
17142.9757 & 303352.7590
\end{array}\right], \\
F 1 & =\left[\begin{array}{cc}
8946.4080 & 152.8018 \\
-88.7054 & 37661.7437
\end{array}\right], & F 2 & =\left[\begin{array}{cc}
-100.1079 & 85.6633 \\
-1447.3920 & 38.6734
\end{array}\right], \\
F 3 & =\left[\begin{array}{cc}
350.8231 & -40.7311 \\
-46.5886 & 5.4090
\end{array}\right], & F 4 & =\left[\begin{array}{cc}
3449.8341 & -375.8000 \\
-500.6067 & 161.2343
\end{array}\right], \\
L & =\left[\begin{array}{cc}
-223754.6705 & 19190.1742 \\
-258925.2826 & 120109.2273
\end{array}\right], & G & =\left[\begin{array}{cc}
37765.6548 & 53117.4530 \\
42764.2230 & 62293.1025
\end{array}\right], \\
K_{o} & =\left[\begin{array}{cc}
-0.5000 & -6.2506 \\
-3.8114 & 6.2506
\end{array}\right], & & \\
\lambda & =816.2566 . & &
\end{aligned}
$$

The stabilizing state feedback gain is then

$$
K=\left[\begin{array}{cc}
-2.2826 & -63.9955 \\
-2.5896 & 45.8611
\end{array}\right]
$$

These results were obtained for

$$
\bar{h}=26.9650 .
$$

The parameter $\bar{h}$ has been found by search, and it is worth noting that this value does not correspond to the maximal value and one can improve this result by choosing adequately the parameters $K_{o}$ and $K_{d_{o}}$. Based on the results of the previous theorem, we conclude that the system under study in this example is robustly stable for all admissible uncertainties.

\section{Conclusion}

This paper has dealt with a class of dynamical linear uncertain systems with multiple time-varying delays in the system state. Delay-dependent sufficient conditions have been developed to check if a system of this class of systems is stable and/or stabilizable. A memoryless state feedback controller with consequent parameters has been used to stabilize the system. The LMI technique is used in all the development. 
152 Stability and stabilizability

\section{References}

[1] E. K. Boukas and Z. K. Liu, Deterministic and Stochastic Time-Delay Systems, Birkhauser, Massachusetts, 2003.

[2] J. Hale, Theory of Functional Differential Equations, Applied Mathematical Sciences, vol. 3, Springer-Verlag, New York, 1977.

[3] A. Hmamed, Further results on the robust stability of linear systems including delayed perturbations, Automatica J. IFAC 33 (1997), no. 9, 1763-1765.

[4] X. Li and C. E. De Souza, Criteria for robust stability of uncertain linear systems with time-varying state delays, IFAC 13th World Congress. Vol 1, International Federation of Automatic Control, California, 1996, pp. 137-142.

[5] Criteria for robust stability and stabilization of uncertain linear systems with state delay, Automatica J. IFAC 33 (1997), no. 9, 1657-1662.

[6] X. Li, M. Fu, and C. E. De Souza, $H_{\infty}$ control and quadratic stabilization of systems with parameter uncertainty via output feedback, IEEE Trans. Automat. Control 37 (1992), 1253-1256.

[7] M. S. Mahmoud, Robust Control and Filtering for Time-Delay Systems, Control Engineering (New York), vol. 5, Marcel Dekker, New York, 2000.

[8] S. I. Niculescu, C. E. De Souza, J. M. Dion, and L. Dugard, Robust stability and stabilization of uncertain linear systems with state delay: single dealy case (i), Proc. IFAC Symposium on Robust Control Design, International Federation of Automatic Control, Rio de Janeiro, 1994, pp. 469-474.

[9] P. Park, Delay-dependent stability criteria for systems with uncertain time-varying delays, to appear.

[10] J.-H. Su, Further results on the robust stability of linear systems with a single time delay, Systems Control Lett. 23 (1994), no. 5, 375-379.

[11] T. J. Su and C. G. Huang, Robust stability of delay dependence for linear uncertain systems, IEEE Trans. Automat. Control 37 (1992), no. 10, 1656-1659.

[12] Y.-J. Sun, J.-G. Hsieh, and H.-C. Yang, On the stability of uncertain systems with multiple timevarying delays, IEEE Trans. Automat. Control 42 (1997), no. 1, 101-105.

[13] S.-S. Wang, B.-S. Chen, and T. P. Lin, Robust stability of uncertain time-delay systems, Internat. J. Control 46 (1987), no. 3, 963-976.

[14] B. G. Xu, On delay-independent stability of large-scale systems with time delays, IEEE Trans. Automat. Control 40 (1995), no. 5, 930-933.

[15] B. G. Xu and Y. Q. Liu, An improved Razumikhin-type theorem and its applications, IEEE Trans. Automat. Control 39 (1994), no. 4, 839-841.

D. Mehdi: Laboratoire d'Automatique et d'Informatique Industielle LAII-ESIP, Université de Poitiers, 40 avenue du Recteur Pineau, 86022 Poitiers Cedex, France

E-mail address: mehdi@esip.univ-poitiers.fr

E. K. Boukas: Départment de Génie Mecanique, École Polytechnique de Montréal, C.P. 6079, Succursale Centre-Ville, Montréal, Québec, Canada H3C 3A7

E-mail address: el-kebir.boukas@polymtl.ca 


\section{Differential Equations \& Nonlinear Mechanics}

\section{An Open Access Journal}

\section{Editor-in-Chief}

K. Vajravelu

USA

Associate Editors

N. Bellomo

Italy

J. L. Bona

USA

J. R. Cannon

USA

S.-N. Chow

USA

B. S. Dandapat

India

E. DiBenedetto

USA

R. Finn

USA

R. L. Fosdick

USA

J. Frehse

Germany

A. Friedman

USA

R. Grimshaw

UK

J. Malek

Czech Republic

J. T. Oden

USA

R. Quintanilla

Spain

K. R. Rajagopal

USA

G. Saccomandi

Italy

Y. Shibata

Japan

Ivar Stakgold

USA

Swaroop Darbha

USA

A. Tani

Japan

S. Turek

Germany

A. Wineman

USA
Website: http://www.hindawi.com/journals/denm/

Aims and Scope

Differential equations play a central role in describing natural phenomena as well as the complex processes that arise from science and technology. Differential Equations \& Nonlinear Mechanics (DENM) will provide a forum for the modeling and analysis of nonlinear phenomena. One of the principal aims of the journal is to promote cross-fertilization between the various subdisciplines of the sciences: physics, chemistry, and biology, as well as various branches of engineering and the medical sciences.

Special efforts will be made to process the papers in a speedy and fair fashion to simultaneously ensure quality and timely publication.

DENM will publish original research papers that are devoted to modeling, analysis, and computational techniques. In addition to original full-length papers, DENM will also publish authoritative and informative review articles devoted to various aspects of ordinary and partial differential equations and their applications to sciences, engineering, and medicine.

\section{Open Access Support}

The Open Access movement is a relatively recent development in academic publishing. It proposes a new business model for academic publishing that enables immediate, worldwide, barrier-free, open access to the full text of research articles for the best interests of the scientific community. All interested readers can read, download, and/or print any Open Access articles without requiring a subscription to the journal in which these articles are published.

In this Open Access model, the publication cost should be covered by the author's institution or research funds. These Open Access charges replace subscription charges and allow the publishers to give the published material away for free to all interested online visitors.

\section{Instructions for Authors}

Original articles are invited and should be submitted through the DENM manuscript tracking system at http://www.mstracking.com/ denm/. Only pdf files are accepted. If, for some reason, submission through the manuscript tracking system is not possible, you can contact denm.support@hindawi.com.

Hindawi Publishing Corporation

410 Park Avenue, 15th Floor, \#287 pmb, New York, NY 10022, USA
HINDAWI 


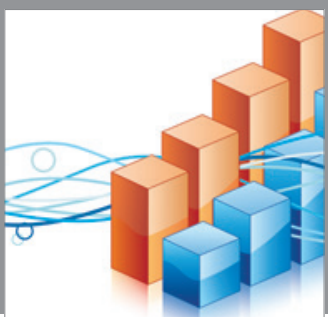

Advances in

Operations Research

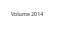

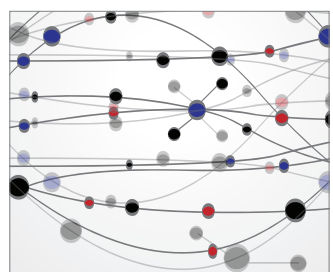

\section{The Scientific} World Journal
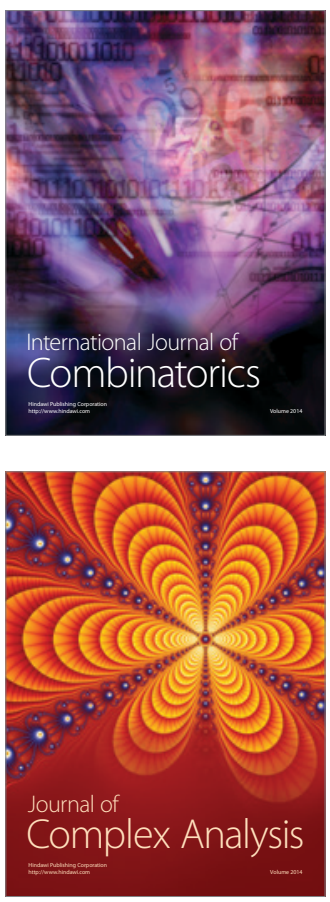

International Journal of

Mathematics and

Mathematical

Sciences
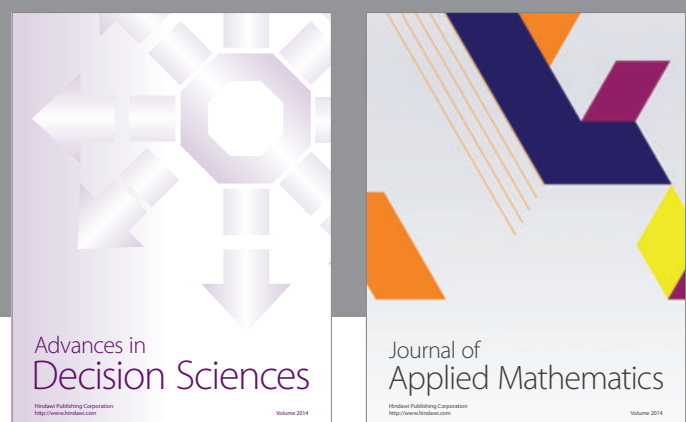

Journal of

Applied Mathematics
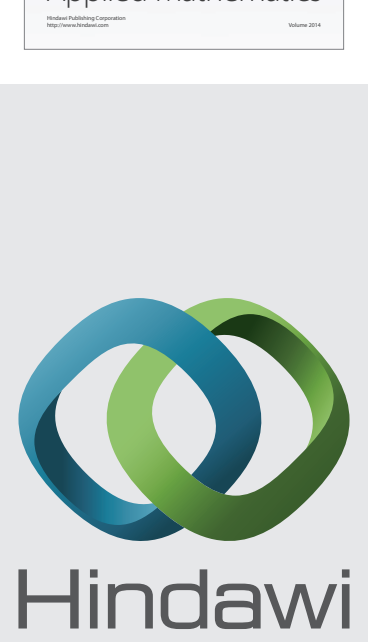

Submit your manuscripts at http://www.hindawi.com
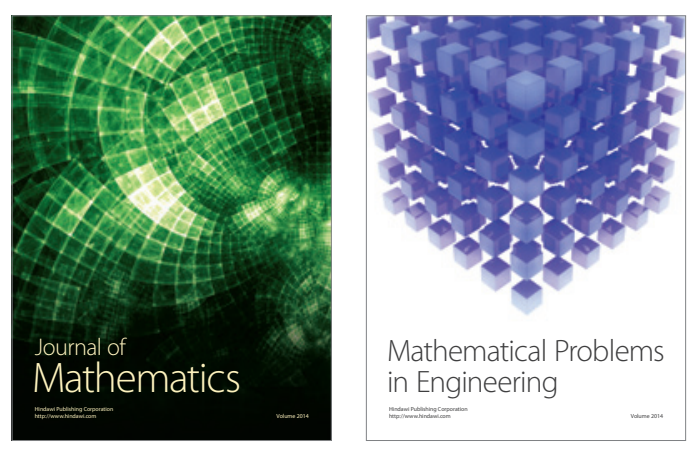

Mathematical Problems in Engineering
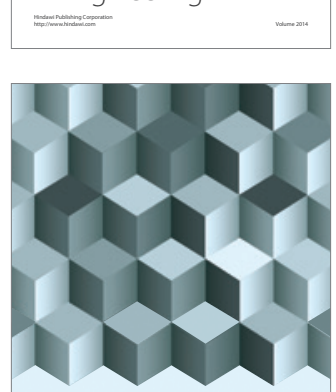

Journal of

Function Spaces
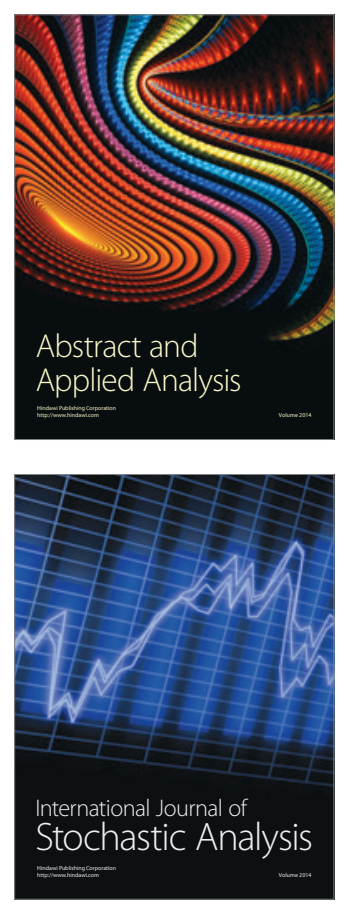

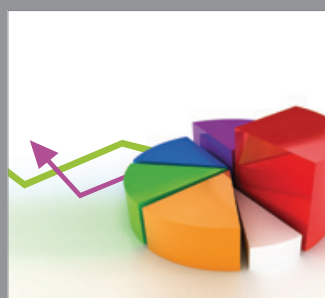

ournal of

Probability and Statistics

Promensencen
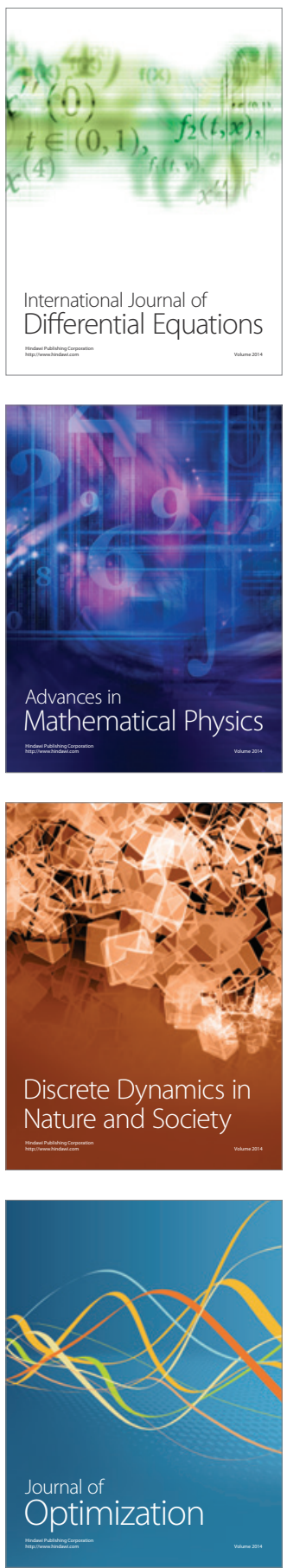